\title{
Test of an Interactive Voice Response Intervention to Improve Adherence to Controller Medications in Adults with Asthma
}

\author{
Bruce G. Bender, PhD, Andrea Apter, MD, Dan K. Bogen, MD, PhD, \\ Perry Dickinson, MD, Larry Fisher, PhD, Frederick S. Wamboldt, MD, and \\ John M. Westfall, MD, MPH
}

Objective: This study was conducted to test the effectiveness of a theory-based interactive voice response (IVR) intervention to improve adherence to controller medications among adults with asthma.

Methods: Fifty participants aged 18 to 65 years who had a physician diagnosis of asthma and a prescription for a daily inhaled corticosteroid, attended a baseline visit and a final visit 10 weeks later. Participants randomized to the intervention group received 2 automated IVR telephone calls separated by one month, with one additional call if they reported recent symptoms of poorly controlled disease or failure to fill a prescription. Calls were completed in less than 5 minutes and included content designed to inquire about asthma symptoms, deliver core educational messages, encourage refilling of inhaled corticosteroid prescriptions, and increase communication with providers. Adherence was tracked during 10 weeks, with objective measures that included either electronic monitors or calculation of canister weight. Participants completed the Asthma Quality of Life Questionnaire, the Asthma Control Test, and the Beliefs in Medications Questionnaire (BMQ) during both visits.

Results: Adherence was 32\% higher among patients in the IVR group than those in the control group $(P=$ .003). A more favorable shift in perception of inhaled corticosteroids was seen on BMQ scores of patients in the IVR group $(P=.003)$, which in turn correlated with degree of adherence change $(\mathrm{r}=0.342 ; P=$ .0152). No differences emerged for the Asthma Quality of Life Questionnaire or Asthma Control Test.

Conclusions: The IVR intervention resulted in a significant increase in adherence to inhaled corticosteroid treatment and improved BMQ scores during the study interval. The association of increased adherence with increased BMQ scores suggests that the intervention succeeded in helping participants adopt a more favorable perception of their controller medication, leading in turn to improved adherence. (J Am Board Fam Med 2010;23:159-165.)

Keywords: Adherence, Interactive Voice Response, Randomized, Asthma, Asthma Inhaler

Patients often do not adhere to treatments for chronic health conditions, with the consequence

This article was externally peer reviewed.

Submitted 8 May 2009; revised 17 July 2009; accepted 21 July 2009.

From the Department of Pediatrics (BGB) and the Department of Psychosocial Medicine (FSW), National Jewish Health, Denver, Colorado; the Department of Medicine, Pulmonary, Allergy Critical Care Division (AA), and the Department of Bioengineering (DKB), University of Pennsylvania, Philadelphia; the Department of Family Medicine, University of Colorado-Denver, Aurora (PD, JMW); and the Department of Family and Community Medicine, University of California, San Francisco (LF)

Funding: This research was conducted with support from the Investigator-Sponsored Study Program of AstraZeneca.

Conflict of interest: none declared.

Corresponding author: Bruce G. Bender, PhD, Professor and Head, Pediatric Behavioral Health, National Jewish Medical and Research Center, 1400 Jackson St., Denver, CO 80206 (E-mail: benderb@njc.org). that many conditions are poorly controlled. In the treatment of asthma, fewer than half of prescribed daily controller medication is typically taken by patients. ${ }^{1,2}$ In a report about refill patterns among 5500 patients with asthma, only $43 \%$ filled their inhaled corticosteroid more than once during 12 months. ${ }^{3}$ In another study, each $25 \%$ decrease in refilled medication was accompanied by a doubling of the hospitalization rate. ${ }^{2}$ Adherence rates among children are similarly poor ${ }^{4,5}$ and remain so even in the face of recent exacerbation. ${ }^{6}$

Considerable attention has been directed at changing health behavior and increasing adherence to treatments for chronic health conditions. The World Health Organization in 2003 published a call for action to improve treatment adherence across diseases, continents, and cultures, ${ }^{7}$ and the 
National Institutes of Health have funded numerous studies targeting strategies to change Americans' health behaviors. Many of the interventions to improve patient adherence have been tested in controlled clinical trials and, although some interventions increase adherence, many are complex, costly, time consuming, and nearly impossible to implement across clinical practice settings. The challenge for health care providers and health behavior professionals is to develop interventions that are cost-effective, easily implemented, and do not place additional burden on clinicians. ${ }^{8}$

Several recent studies have examined the utilization of advances in information technology to communicate with patients and change their behavior. Speech recognition and text messaging programs have been designed to promote diabetes and asthma self management. ${ }^{9-11}$ E-mail and webbased interventions have targeted improved adherence to antihypertensive medications ${ }^{12}$ as well as smoking cessation ${ }^{13}$ and weight loss programs. ${ }^{14}$ Although such interventions have been developed and described, few have been tested in randomized clinical trials.

The clever application of information technology to patient care may not translate into behavior change. Interventions must be based on tested theories of health behavior to ensure success. One particularly helpful health behavior theory is the benefit-risk model, ${ }^{15}$ which posits that the probability of engaging in health-promoting behaviors depends on the person's perception of risk and benefit related to the behavior and its health consequence.

In the case of patients with chronic illnesses, the decision about whether or not to adhere to a treatment plan is largely influenced by their perception of the risks associated with the disease and the risks and benefits introduced by the treatment. ${ }^{16}$ Following the benefit-risk model, a change in asthma treatment adherence behavior will occur when patient perceptions of the relative benefits of using asthma controller medication have changed so that the patient adopts a more favorable view of the benefits. ${ }^{17}$ When adult asthma patients were asked to rate advantages and disadvantages of using asthma medication using the Beliefs in Medication Questionnaire (BMQ), a lower benefit-to-risk differential predicted lower controller medication adherence among adults ${ }^{18}$ and children with asthma. ${ }^{19}$ Recognizing the potential efficiency of using information technology to change patient be- havior when guided by sound health behavior theory, the present study was designed to test the use of advanced speech recognition technology to strategically alter patients' perceptions of the benefit of their controller medication and, consequently, to improve the successful management of their disease.

\section{Methods \\ Participants}

Fifty 18 - to 65 -year-old adults who had physiciandiagnosed asthma for which they were prescribed daily inhaled corticosteroid treatment participated. Participants were recruited through newspaper advertising and in cooperation with community allergy practices and they received $\$ 25$ for each completed study visit. Exclusion criteria included (1) any significant disease or disorder that, in the opinion of the investigator, might influence the results of the study or the patient's ability to participate in the study (this included other chronic health disorders, current substance abuse or dependence, mental retardation, or psychiatric disorder); and (2) current participation in any other asthma-related research or clinical trial.

\section{Design and Sample Size}

This was a 10 -week randomized clinical trial comprising 2 groups of patients. Using results from a previous 10 -week study that tested an adherence intervention among patients with asthma, ${ }^{20}$ power and sample size calculations indicated that 25 participants in each group would provide $75 \%$ power to detect a group difference of $36 \%$.

\section{Procedures}

This was a single-site study conducted at National Jewish Health in Denver, Colorado. National Jewish Health is a tertiary care center that focuses on the treatment of children and adults with respiratory, allergic, and immune disorders. Each participant attended an initial baseline visit and, if eligible, provided informed consent before being randomized to either the intervention or control group. A randomization table generated before study initiation determined group assignment by order of entry into the study. The investigators remained blind to treatment until the final data set was completed. All participants completed questionnaires at baseline and during a final visit that included: 
1. Astbma Control Test (ACT). The ACT is a 5 -item questionnaire completed by the patient that assesses the level of symptom control during the previous 4 weeks. ${ }^{21}$ Higher scores indicate better control of asthma symptoms.

2. Asthma Quality of Life Questionnaire (AQLQ). The AQLQ is a disease-specific quality of life questionnaire that measures both the physical and emotional impact of asthma with 32 questions representing 4 domains (symptoms, activity limitation, emotional function, and environmental stimuli). ${ }^{22}$ Higher scores in each domain indicate better quality of life.

3. Beliefs about Medications Questionnaire. The BMQ includes 5 items that assess negative beliefs about medications ("I worry about the long-term effects of this medicine.") and 5 items that assess positive beliefs ("This medicine protects me from becoming worse."). Each item is rated by the patient on a Likerttype scale from 1 (strongly agree) to 5 (strongly disagree). ${ }^{23}$ BMQ scores from the baseline and 10 -week visits were calculated by subtracting the negative index total from the positive index total. Hence, scores above 0 indicated more positive beliefs and scores below 0 indicated more negative beliefs.

One of 3 electronic tracking devices-the Metered-Dose Inhaler Log, the Doser-Clinical Trials version, or the Diskus Adherence Monitor-was attached to each patient's inhaler for the 10-week study interval and was collected during the final visit. For patients taking combination budesonide/formoterol (to which an electronic device cannot be attached), the change in canister weight between visits was used to calculate the number of puffs taken from the inhaler. Accuracy of all 4 objective adherence measurements has been established. ${ }^{4,24,25}$ This study was approved by the Institutional Review Board of National Jewish Health.

\section{Interactive Voice Response Intervention.}

The interactive voice response (IVR) system consisted of several technologies working together to schedule, make, receive, and record automated phone calls. Included were (1) a server that controlled all core system functionality, (2) a database used for storage of all call information, (3) IVR software, which controlled call flow and data input functions, (4) application software that was used to schedule phone calls, and (5) a telephony card used to connect to the phone system. IVR systems can be programmed to make and receive automated telephone calls and have the capability to ask questions and give information, which tailors IVR responses to the answers given by the respondent using their telephone keypad. The content of an IVR call depends on the purpose of the call and the behavior-change strategy adopted by the project. For this project the IVR intervention was based on the benefit-risk model of health behavior, which assumes that the patient's perceptions of the relative benefits of using asthma controller medication must be addressed before a change in adherence behavior will occur. ${ }^{26}$

To alter the patient's perception of the relative benefits of taking their controller medication, content of the IVR intervention was developed to increase patient communication with providers, inquire about asthma symptoms, deliver core educational messages, encourage patients to ask questions of asthma care providers, and encourage refilling of inhaled corticosteroid (ICS) prescriptions. After the drafting of an initial IVR script was completed, 4 focus groups were convened, each of which consisted of 8 to 10 patients or family members of patients with asthma. The goal, design, and planned content of the IVR project were described to the focus groups, and feedback from focus group members was then used to refine content and develop final script. After programming of the IVR, a test phase was conducted, during which 50 calls were completed and participant responses were solicited. None of the participants in this test phase were participants in the randomized trial. Final refinements included wording changes and adjustment of the timing of content in the calls as well as time between calls.

Each patient received at least 2 calls separated by 1 month. Calls were programmed to reach out at several time points throughout the day and evening until the participant answered. If an answering machine was reached, a toll-free number was provided, which the participant could use to call back. When a call connection was completed, the IVR call identified itself as coming from the Denver Interactive Asthma Learning System program and verified that the correct person had been called (Figure 1). Content of the call then included an explanation of how the call works followed by 3 questions inquiring whether during the previous 
Figure 1. Content of the interactive voice recognition call.

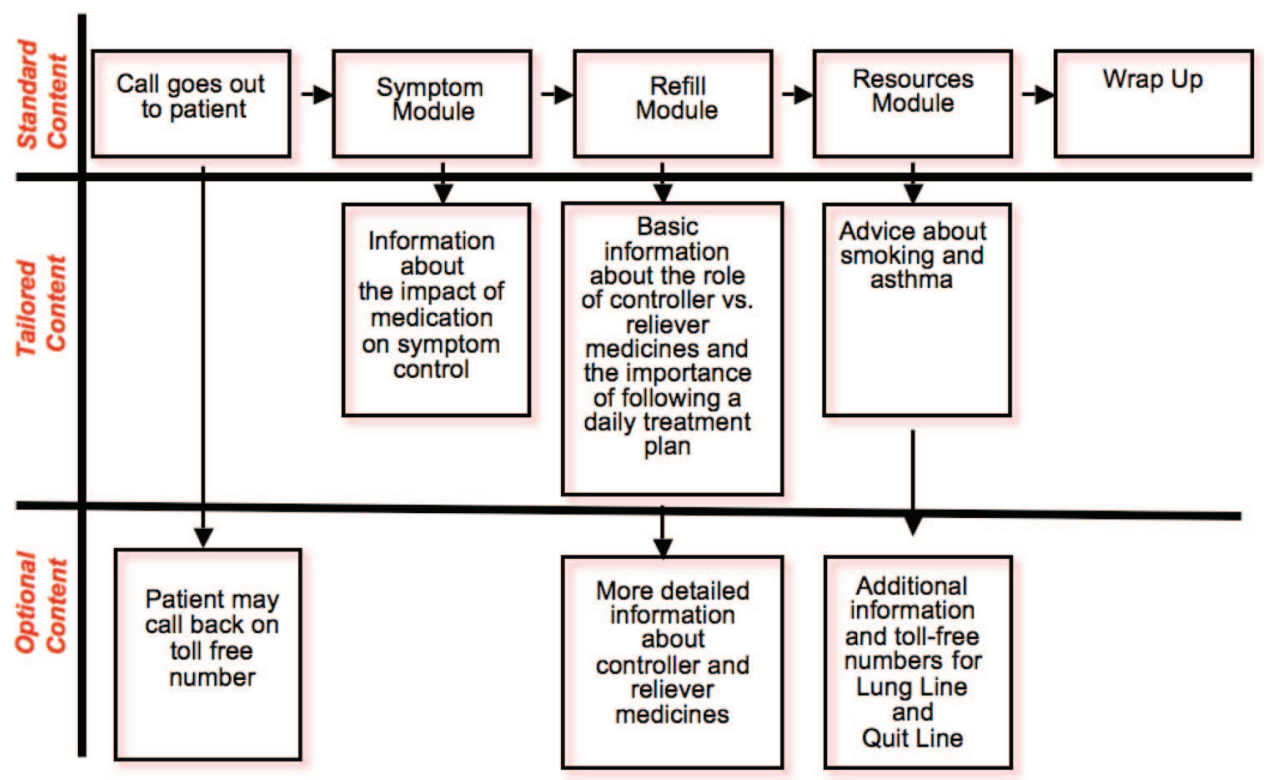

week the participant had been awakened at night, had limited their activities, or had used their rescue inhaler more than twice because of asthma symptoms (symptom module). Participants who responded affirmatively to any of the 3 questions were told that daily use of their controller medication should help prevent such symptoms and were advised to discuss the symptoms with their physician. All participants also listened to a short module about the benefits of their asthma medication and were asked about whether they were filling and using their medication, with IVR responses tailored to specific participant responses (refill module). Finally, participants were informed about the Lung Line, a free telephone service staffed by nurses capable of answering most questions about asthma, and about the Colorado Quit Line, offering free telephonebased tobacco cessation intervention (resources module). Finally, participants were thanked for their participation. Participants who reported symptoms during either of the 2 calls or who indicated that they did not intend to refill their medication were scheduled to receive a third call 2 weeks later. No participant received more than 3 calls. Participants in the control group received no calls.

\section{Data Analyses}

All statistical analyses were performed with JMP software (SAS Institute, Inc., Cary, North Carolina). An analysis of variance using the Dunnett test was used for comparing demographic data, adherence, and BMQ and ACLQ scores. Percent adherence was determined by dividing the number of inhaler puffs taken by the number of puffs prescribed to be taken each day and then averaged over the 10-week interval. Final scores from the AQLQ and BMQ were calculated by subtracting the baseline score from the final score. Bivariate fit modeling was used to assess potential correlations between adherence level and changes in medication beliefs.

\section{Results}

The treatment and control groups did not differ in demographic data, including age, gender frequencies, or racial group frequencies, although trends suggested more men and Hispanics and fewer Asian participants in the control group (Table 1). Mean ICS adherence was higher in the group receiving IVR intervention than in the control group by a margin of $64.5 \%$ to $49.1 \%(\mathrm{~F}=9.66 ; P=.0032)$. The 2 groups also differed on the BMQ, with the group receiving IVR intervention demonstrating a greater upward shift in positive medication beliefs $(\mathrm{F}=7.905 ; P=.007)$. No mean group differences emerged for any of the AQLQ or ACT scores (Table 2). In the bivariate fit of means model, significant correlation was found between adherence and BMQ scores, such that adherence in- 
Table 1. Comparisons between Treatment and Control Groups Indicating No Differences in Age, Gender, or Race

\begin{tabular}{lllc}
\hline & $\begin{array}{c}\text { Treatment } \\
\text { Group }(\mathrm{n} \\
=25)\end{array}$ & $\begin{array}{c}\text { Control } \\
\text { Group } \\
(\mathrm{n}=25)\end{array}$ & $P$ \\
\hline Age, y (mean [SD]) & $39.6(12.8)$ & $43.5(14.3)$ & $.137^{*}$ \\
Gender (\% male) & 40 & 32 & $.556^{\dagger}$ \\
Race (\%) & & & .301 \\
White & 56 & 60 & \\
Hispanic & 24 & 12 & \\
African American & 20 & 20 & \\
Asian & 0 & 8 & \\
\hline
\end{tabular}

*Dunnett.

${ }^{+} \chi^{2}$.

creased as BMQ scores increased $(\mathrm{r}=0.342 ; P=$ $.0152)$.

\section{Discussion}

The IVR intervention improved adherence by $32 \%$ during the 10-week study interval. Use of communication technology to change health behavior is an emerging field of investigation, and not all attempts to change behavior with such technology have been successful. One successful IVR program improved smoking cessation by 33\% compared with a control group. ${ }^{9}$ Other voice recognition technologies have recently been used to promote treatment adherence in asthma ${ }^{10}$ and diabetes self-management. ${ }^{11}$ In addition, text messaging has been used to support adolescents with asthma ${ }^{27}$ and diabetes. ${ }^{28}$ Email interventions have been introduced to improve blood pressure treatment adherence, ${ }^{12}$ and a variety of web-based interventions have been used to improve smoking cessation, ${ }^{13}$ weight loss, ${ }^{14}$ hypertension, ${ }^{12}$ and chronic obstructive pulmonary disease. ${ }^{29}$ Early reports from some communication technology projects have described patient response to using the telecommunication technology but did not report behavior or disease outcomes. ${ }^{30,31}$ The application of such telecommunication technology in primary care does not relieve the physician of the responsibility to communicate effectively with patients to encourage treatment adherence. Thoughtful communication that includes sensitivity to patient concerns and preferences and a willingness to negotiate treatment goals and plans is a powerful strategy that will lead to greater adherence and treatment success. ${ }^{32}$ However, employment of automated communication technology can complement the physician's efforts and increase successful disease self-management while protecting clinicians' time.

Variability in the success of communication technology interventions may reflect, in part, differences in the degree to which patients' benefitrisk perceptions were altered. ${ }^{26}$ The IVR intervention in the present study was designed to change patients' perception of the relative benefits of adhering to a daily ICS treatment plan. Three modules were introduced during each call to inquire about symptoms, prescription filling, and medication use and to direct participants to other sources of information. Both the symptom and refilling modules were tailored so that advice given to participants depended on the answers given by participants. For example, participants who reported the occurrence of multiple symptoms during the previous week were told that their controller medication could help prevent such symptoms and were

Table 2. Comparisons between Treatment and Control Groups Indicating Change in Adherence and Medication Beliefs from Baseline to Final Visit

\begin{tabular}{lccc}
\hline & Treatment Group $(\mathrm{n}=25)$ & Control Group $(\mathrm{n}=25)$ & $P$ \\
\hline Percent adherence & $64.5(17.2)$ & $49.1(16.8)$ & .003 \\
Belief in Medications Questionnaire & $0.248(1.07)$ & $-0.508(0.913)$ & .007 \\
Asthma Quality of Life Questionnaire & & & \\
$\quad$ Activity & $-0.151(0.92)$ & $-0.381(1.06)$ & .172 \\
Symptoms & $-0.247(1.13)$ & $-0.380(1.37)$ & .823 \\
Emotions & $-0.128(0.24)$ & $-0.376(1.30)$ & .488 \\
Environment & $-0.152(0.84)$ & $-0.360(1.23)$ & .486 \\
$\quad$ Total & $-0.152(0.92)$ & $-0.381(1.06)$ & .419 \\
Asthma Control Test & $-1.120(3.90)$ & $-1.840(4.14)$ & .530 \\
\hline
\end{tabular}

All values provided as mean (SD). 
given an opportunity to learn more about the differences between controller and rescue medications. As directed by focus group feedback, information and advice was brief, allowing the call to be completed in less than 5 minutes. Significant changes in BMQ scores toward more positive views of medication among patients in the intervention group, as well as the correlation between BMQ scores and adherence, suggest that change of perceptions of patients in the intervention group helped to improve adherence. It is not surprising that, across a 10 -week trial, adherence and beliefs about ICS changed but asthma control reflected in ACT scores did not because these outcomes typically require a greater time to response. However, a $32 \%$ increase in the use of inhaled corticosteroids is a large change and, based on other large studies about the impact of these medications on asthma control, would be likely to significantly improve disease self-management and symptom control while reducing frequency of $\beta$-agonist use and risk of hospital visits or oral steroid requirement. ${ }^{2,33-35}$ Future, longer studies are needed to demonstrate that the improved adherence achieved by the IVR protocol actually improves asthma symptoms and quality of life. The successful application of this program to patients with asthma may help promote more effective self-management among patients who have other chronic conditions.

\section{Limitations}

The limitations of this study should be considered. Behavior change attained during any controlled study may not easily translate into broad clinical practice. Change in adherence behavior achieved during the study may not be sustained after the program ends. An automated program may not adequately address the concerns of all groups of patients. Future research applications will require testing in a variety of practice settings, including health maintenance organizations, community health clinics, and rural practices. This study did not collect patient information regarding the time since diagnosis, previous adherence to medication, or symptom level. To better target the recipients of such an intervention, it may be helpful in future investigations to determine whether newly diagnosed or nonadherent patients respond differently to this intervention than those who have longer asthma histories or who have been more adherent to treatment in the past. In addition, investigations should focus on the adherence-sustaining capacity of a long-term IVR program, the cost of which may be relatively low once the program is established. Finally, new approaches to altering patients' perceptions about the benefits and necessity of adherence to prescribed self-management practices may be adopted into communication technology protocols to more effectively change health behavior, including tailoring interventions to address the concerns and perceptions of subpopulations of asthma patients, including adolescents, seniors, and low-income urban and rural patients.

\section{Conclusion}

Communication technology cannot replace the physician-patient interaction as the primary source of information about and motivation toward effective self-management of disease. It does, however, offer an important opportunity to extend physician-patient communication, help increase patient motivation, and improve cost efficiency while increasing patients' ability to manage their disease. IVR and other communication technologies show particular promise for rural health care, where patients may live in remote areas and require long travel times to see a provider and who therefore may not receive the support necessary to encourage optimal self-management of their diseases. For these reasons, new technologies will continue to be examined for their potential to improve disease control among patients with chronic illnesses.

\section{References}

1. Stempel D, Roberts C, Stanford R. Treatment patterns in the months prior to and after asthma-related emergency department visit. Chest 2004;126:75-80.

2. Williams L, Pladevall M, Xi H. Relationship between adherence to inhaled corticosteroids and poor outcomes among adults with asthma. J Allergy Clin Immunol 2004;114:1288-93.

3. Bender B, Pedan A, Varasteh L. Adherence and persistence with fluticasone propionate/salmeterol combination therapy. J Allergy Clin Immunol 2006; 118:899-904.

4. Bender B, Bartlett S, Rand C, Turner C, Wamboldt $\mathrm{F}$, Zhang L. Impact of interview mode on accuracy of child and parent report of adherence with asthma controller medication. Pediatrics 2007;120:e471-7.

5. Walders N, Kopel S, Koinis-Mitchell D, McQuaid E. Patterns of quick-relief and long-term controller medication use in pediatric asthma. J Pediatr 2005; 146:177-82.

6. Cooper W, Hickson G. Corticosteroid prescription 
filling for children covered by Medicaid following an emergency department visit or a hospitalization for asthma. Arch Pediatr Adolesc Med 2001;115:1111-5.

7. World Health Organization. Chronic diseases and health promotion. Adherence to long-term therapies evidence for action. Available at http://www.who.int/ chp/knowledge/publications/adherence_report/en/ index.html. Accessed 20 January 2010.

8. Bender B. Unmet needs in the long-term maintenance treatment of asthma - addressing treatment adherence. US Respiratory Disease 2009;4:20-3.

9. Reid R, Pipe A, Quinlan B, Oda J. Interactive voice response telephony to promote smoking cessation in patients with heart disease: a pilot study. Patient Educ Couns 2007;66:319-26.

10. Vollmer W, Kirshner M, Peter S. Use and impact of an automated telephone outreach system for asthma in a managed care setting. Am J Manage Care 2006; 12:725-33.

11. Goldman R, Sanchez-Hernandez M, Ross-Degnan D, Piette J, Trinacty C, Simon S. Developing an automated speech-recognition telephone diabetes intervention. J Qual Health Care 2008;20:264-70.

12. Green B, Ralston J, Fishman P. Electronic communications and home blood pressure monitoring (eBP) study design delivery, and evaluation. Contem Clin Trials 2008;29:376-95.

13. Stoddard J, Augustson E, Moser R. Effect of adding a virtual community (bulletin board) to smokefree. gov: randomized controlled trial. J Med Internet Res 2008; 10:e53.

14. Brantley P, Appel L, Hollis J. Design considerations and rationale of a multi-center trial to sustain weight loss: the Weight Loss Maintenance Trial. Clin Trials 2007;5:546-56.

15. Horne R, Weinman J. Patients' beliefs about prescribed medicines and their role in adherence to treatment in chronic physical illness. J Psychosom Res 1999;47:555-67.

16. Rosenstock I. Historical origins of the health belief model. Health Education Monographs 1974;2:328-35.

17. Horne R, Weinman J. Patients' beliefs about prescribed medicines and their role in adherence to treatment in chronic physical illness. J Psychosom Res 1999;47:555-67.

18. Horne R, Weinman J. Self-regulation and self-management in asthma: Exploring the role of illness perceptions and treatment beliefs in explaining nonadherence to preventer medication. Psychology and Health 2002;17:17-32.

19. Conn K, Halterman J, Fisher S, Yoos H, Chin N, Szilagyi P. Parental beliefs about medications and medication adherence among urban children with asthma. Ambul Pediatr 2005;5:306-10.

20. Onyirimba F, Apter A, Reisine S, et al. Direct clinician-to-patient feedback discussion of inhaled steroid use: its effect on adherence. Ann Allergy Asthma Immunol 2003;90:411-5.
21. Nathan R, Sorkness C, Kosinski M, et al. Development of the asthma control test: a survey for assessing asthma control. J Allergy Clin Immunol 2004; 113:59-65.

22. Juniper E, Guyatt G, Epstein R, Ferrie P, Jaeschke $\mathrm{R}$, Hiller T. Evaluation of impairment of healthrelated quality of life in asthma: development of a questionnaire for use in clinical trials. Thorax 1992; 47:76-83.

23. Horne R, Weinman J. Self-regulation and self-management in asthma: exploring the role of illness perceptions and treatment beliefs in explaining nonadherence to preventer medication. Psychol Health 2002;17:17-32.

24. Bender B, Wamboldt F, O'Connor S, et al. Measurement of children's asthma medication adherence by self-report, mother report, canister weight, and Doser CT. Ann Allergy Asthma Immunol 2000;85: 416-21.

25. Bogen D, Apter A. An adherence logger for a drypowder inhaler: a new device for medical adherence research. J Allergy Clin Immunol 2004;114:863-8.

26. Horne R. Compliance, adherence, and concordance implications of asthma treatment. Chest 2006;130(1 Suppl):65S-72S.

27. Neville R, Greene A, McLeod J, Tracey A, Surie J. Mobile phone text messsaging can help young people manage asthma. BMJ 2002;14:600.

28. Franklin V, Waller A, Pagliari C, Greene S. A randomized controlled trial of Sweet Talk, a text-messaging system to support young people with diabetes. Diabet Med 2006;23:1332-8.

29. Borycki E, Kushniruk A. Development of a virtual selfmanagement tool for COPD patients: towards a user needs ontology. AMIA Annu Symp Proc 2007:879.

30. Reidel K, Tamblyn R, Patel V, Huang A. Pilot study of an interactive voice response system to improve medication refill compliance. BMC Med Inform Decis Mak 2008;8:46.

31. Mollen B, Holbrook AM, Keshavjee K, et al. Automated telephone reminder messages can assist electronic diabetes care. J Telemed Telecare 2008;14:32-6.

32. Bender B, Rankin A, Tran ZV, Wamboldt FS. Brief interval telephone surveys of medication adherence and asthma symptoms in the Childhood Asthma Management Program Continuation Study. Ann Allergy Asthma Immunol 2008;101:382-386.

33. Adams R, Fuhlbrigge A, Finkelstein J. Impact of inhaled anti-inflammatory therapy on hospitalizations and emergency department visits for children with asthma. Pediatrics 2001;107:706-11.

34. Sullivan S, Buston M, Anderson L. Cost-effective analysis of early intervention with budesonide in mild persistent asthma. J Allergy Clin Immunol 2003;112:1229-36.

35. Donahue J, Weiss S, Livingston J, Goetsch M, Greineder D, Platt R. Inhaled steroids and the risk of hospitalization for asthma. JAMA 1997;277:887-91. 